\title{
Evaluation de la disponibilité des personnels qualifiés en santé maternelle et néonatale à Madagascar
}

\author{
Authors: \\ Sandrine Andriantsimietry ${ }^{1}$ \\ JeanPierre Rakotovao ${ }^{1}$ \\ Eliane R. Ramiandrison ${ }^{1}$ \\ Haja Andriamiharisoa \\ Eric M.R. Razakariasy ${ }^{1}$ \\ Rachel Favero ${ }^{2}$ \\ Eva Bazant ${ }^{3}$ \\ Patricia Gomez ${ }^{4}$ \\ Blami Dao ${ }^{4}$

\begin{abstract}
Affiliations:
${ }^{1}$ Maternal and Child Surviva Program (MCSP), Jhpiego,

Madagascar
\end{abstract} \\ ${ }^{2}$ Maternal and Child Surviva \\ Program (MCSP), Jhpiego, \\ Baltimore, MD, United States \\ ${ }^{3}$ Jhpiego, Washington DC, \\ United States \\ ${ }^{4} J$ hpiego, Baltimore, United \\ States \\ Correspondence to \\ Sandrine Andriantsimietry \\ Email: \\ sandrine.andriantsimietry@ \\ jhpiego.org

\section{Postal address:} \\ Immeuble Santa Lot II \\ 3ème étage Antanimena, \\ Antananarivo 101 \\ Madagascar \\ Dates: \\ Received: 15 May 2015 \\ Accepted: 28 July 2015 \\ Published: 12 Oct. 2015 \\ How to cite this article: \\ Andriantsimietry, S., \\ Rakotovao, J., Ramiandrison \\ E.R., Andriamiharisoa, H., \\ Razakariasy, E.M.R., \\ Favero, R. et al., 2015 \\ 'Evaluation de la disponibilité \\ des personnels qualifiés \\ en santé maternelle et \\ néonatale à Madagascar', \\ African Evaluation Journal \\ 3(2), Art. \#156, 8 pages. \\ http://dx.doi.org/10.4102/ \\ aej.v3i2.156 \\ Read online:

A Madagascar, la situation de la santé maternelle et néonatale demeure inquiétante avec un ratio de mortalité maternelle de 478 pour 100000 naissances vivantes et des taux de mortalité néonatale et infantile de 26 pour 1000 naissances vivantes et de 42 pour 1000 naissances vivantes, pour les enfants âgés de moins d'un mois et les enfants âgés de moins d'un an respectivement. Plusieurs facteurs pourraient expliquer cet alarmant état de santé maternelle et néonatale, y compris la pénurie de personnel de santé qualifié. Etant donné que plusieurs interventions ont été réalisées pour l'atteinte des objectifs 4 et 5 de l'OMD et qu'elles n'ont pas été fructueuses, il est pertinent d'évaluer la disponibilité des agents de santé qualifiés pour offrir les services de santé maternelle et néonatale dans les établissements sanitaires publics. L'enquête transversale a utilisé et adapté l'outil SARA (Service Availability and Readiness Assessment) de l'Organisation Mondiale de la Santé dans les 52 établissements sanitaires publics, dans 15 des 22 régions de Madagascar. Les données ont été collectées sur tablette avec le logiciel CommCare ODK@ et analysées par niveau des établissements sanitaires avec le SPSS20.0. Le médecin généraliste est absent dans 14\%des hôpitaux de référence et $22 \%$ des centres de santé primaire. Le nombre médian de gynécologue obstétricien dans les hôpitaux pour pratiquer les opérations césariennes est de 1 . Seulement $20 \%$ des 15 régions enquêtées ont une proportion élevée (> 80\%) de personnels qualifiés en santé maternelle et néonatale dans les établissements sanitaires publics. Dans l'optique d'une réduction tangible des mortalités maternelles et néonatales, l'on devrait renforcer la capacité de de ressources humaines et leur disponibilité pour offrir les services de santé maternelle et néonatale dans les établissements sanitaires publics.

Evaluation of the availability of the personnel qualified in maternal and neonatal health in Madagascar.

In Madagascar, the position of maternal and neonatal health is worrying with a maternal mortality ratio of 478 per 100000 live births and a neonatal and infantile mortality of 26 per 1000 live births and 42 per 1000 live births, for children younger than one month and those younger than one year, respectively. Several factors could explain this alarming maternal and neonatal state of health, including the lack of qualified health workers. Considering that several interventions were carried out to reach aims 4 and 5 of WHO and that they were not successful, it is appropriate to assess the availability of qualified health workers to provide maternal and neonatal health services in public health facilities. The cross-sectional survey used and adapted the SARA tool (Service Availability and Readiness Assessment) of the World Health Organisation in the 52 public health facilities, in 15 of the 22 regions of Madagascar. The data was collected on tablets with CommCare ODK@ software and analysed by level of health facilities with SPSS20.0. There is no general practitioner in $14 \%$ of the reference hospitals and $22 \%$ of primary health centres. The median number of obstetrician-gynaecologist in hospitals that can do caesarean sections is 1 . Only $20 \%$ of the 15 regions surveyed have a high proportion of qualified personnel $(>80 \%)$ in maternal and neonatal health in public health facilities. To achieve a tangible reduction of maternal and neonatal mortality, the human resource capacity and availability must be strengthened to provide maternal and neonatal services in public health facilities.

\section{Introduction}

\section{Contexte}

Entre les années 1990 et 2013, le taux de mortalité maternelle mondial a diminué de 2,6\% par an et pourtant, plus de la moitié des décès maternels globaux surviennent en Afrique subsaharienne (WHO 2014a). A Madagascar, le taux de mortalité maternelle est stationnaire depuis 10 ans et 
la dernière enquête, en 2013 a donné un chiffre de 478 pour 100000 naissances vivantes. En outre, le taux de mortalité néonatale auprès des enfants âgés de moins d'un mois est de 26 pour 1000 naissances vivantes tandis que le taux de mortalité auprès des enfants âgés de moins de 12 mois est de 42 pour 1000 naissances vivantes et celui auprès des enfants de moins de 5 ans est de 62 pour 1000 naissances vivantes (INSTAT Madagascar 2013a). Ces chiffres se trouvent encore éloignés des cibles du pays pour les Objectifs 4 et $5 \mathrm{du}$ Millénaire pour le Développement (OMD) pour la réduction de la mortalité des enfants de moins de 5 ans et l'amélioration de la santé maternelle qui sont respectivement de 21 pour 1000 naissances vivantes pour le taux de mortalité néonatale, de 53 pour 1000 naissances vivantes pour le taux de mortalité des enfants de moins de 5 ans et de 122 pour 100000 naissances vivantes pour le ratio de mortalité maternelle (INSTAT Madagascar 2013a).

L'évaluation des ressources humaines qui contribuent activement dans le système de santé est rarement réalisée à l'échelle mondiale, particulièrement dans les pays en développement. Pourtant, les ressources humaines pour la santé constituent un des déterminants clés du système de santé. Au cours de la dernière décennie, l'on a commencé à prendre conscience de l'impact de la pénurie en agents de santé dans les pays ayant de faibles valeurs des indicateurs de santé sur leur capacité à combattre les maladies et à offrir des interventions qui sauvent des vies (Chen et al. 2004; Narasimhan et al. 2004; WHO 2006).

La pénurie mondiale en agents de santé est estimée à environ 4, 3 millions, et les pays les plus touchés sont ceux qui portent de lourds fardeaux de maladies. Selon l'OMS, l'actuel personnel de santé dans les pays les plus affectés en Afrique subsaharienne nécessiterait une augmentation de 140\% pour permettre l'atteinte des Objectifs du Millénaire pour le Développement (UNICEF 2009). En effet, l'on signale que plusieurs régions ne pourront pas atteindre leurs objectifs du millénaire pour leur développement, particulièrement la région de l'Afrique subsaharienne (The Lancet Commissions 2010).

Dans l'ensemble, cette étude se proposait d'évaluer le statut des offres de service en santé maternelle et néonatale dans les établissements sanitaires publics. A travers cette étude, l'objectif du présent manuscrit est d'évaluer la disponibilité des agents de santé qualifiés, c'est-à-dire ayant eu des récentes mises à jour sur leurs connaissances et leurs compétences techniques, pour offrir les services de santé de la mère et de l'enfant dans les établissements sanitaires publics de 15 régions de Madagascar.

\section{Revue de la littérature}

Peu de littérature traite les questions de ressources humaines au service de la santé à Madagascar. La vulnérabilité de la capacité du système de santé du pays à atteindre les objectifs pour la réduction de la mortalité maternelle a été signalée depuis 2010, et la contribution active des agents de santé travaillant dans le secteur public et le secteur privé serait une stratégie pour accroître les chances d'atteindre les Objectifs du Millénaire pour le Développement (Harimanana, Barennes \& Reinharz 2011). Au cours d'une enquête en 2010 sur la qualité des soins dans le domaine de la santé de la mère et du nouveau-né, réalisée par le programme intégré de santé maternelle et infantile (PMI, pour les anglo-saxons: MCHIP), il a été constaté que les connaissances des prestataires sont particulièrement faibles, notamment sur les complications à l'origine des décès maternels ainsi que leurs prises en charge. En revanche, les prestataires de santé avaient de meilleures connaissances sur les soins du nouveau-né (MCHIP Madagascar-Jhpiego 2011).

L'évaluation du volet de la disponibilité des ressources humaines, composées d'agents de santé qualifiés, requise pour offrir les services de santé aux patients au niveau des établissements sanitaires a été effectivement délaissée au profit des services eux-mêmes. Pourtant, ces services ne seraient effectifs pour sauver des vies et améliorer la santé des clients des établissements sanitaires qu'avec l'implication d'agents de santé qualifiés. Par ailleurs, ces données pourraient être utilisées pour appuyer et identifier les stratégies effectives pour la réduction des mortalités maternelles et néonatales. En effet, certains auteurs ont auparavant attribué les décès maternels et infantiles dans le contexte de l'Afrique subsaharienne à la déficience en agents de santé qualifiés dans les établissements sanitaires (Anand \& Bärnighausen 2004; Gerein, Green \& Pearson 2006). En outre, la compétence des agents de santé, quand bien même ces agents seraient insuffisants en nombre, permettrait aux pays d'atteindre les Objectifs du Millénaire pour le Développement relatifs à la santé (Fulton et al. 2011), et le renforcement continu des connaissances et des compétences techniques des agents de santé se traduirait en une amélioration de la santé maternelle et néonatale (Ariff et al. 2010).

Cette évaluation, réalisée au début du programme pour la survie de la mère et de l'enfant (PSME, pour les anglo-saxons: MCSP) au dernier semestre 2014, confirme la faiblesse des ressourceshumainespourl'offredeservicesensantématernelle et néonatale dans les établissements sanitaires publics qui serait un des facteurs nuisant à l'atteinte des Objectifs du Millénaire pour le Développement à Madagascar. Dans cette étude, nous avons utilisé l'instrument de collecte de données SARA (Service Availability and Readiness Assessment) développé conjointement par l'Organisation Mondiale de la Santé et l'Agence Internationale de Développement des USA (USAID). Cet outil est axé sur 3 domaines principaux: la disponibilité des services, la capacité opérationnelle générale des services et la disponibilité et capacité opérationnelle des services de santé spécifiques concernant les personnels formés, les documents, les équipements, les médicaments et produits de santé. L'outil SARA permet en effet, de générer des informations objectives sur la capacité et l'habilité des établissements sanitaires à offrir les services de santé, et comprend une large section sur les prestataires de santé. Dans l'outil de collecte de données SARA, un agent de santé qualifié se définit par une personne ayant eu une formation 
ou une mise à jour récente, de ses connaissances et de ses compétences techniques, au cours des 2 dernières années (O'Neill et al. 2013; WHO 2013).

\section{Méthodes}

La présente étude a été réalisée dans 15 (Alaotra Mangoro, Amoron'i Mania, Analamanga, Atsinanana, Atsimo Andrefana, Boeny, Diana, Haute Matsiatra, Ihorombe, Melaky, Menabe, Sofia, Sava, Vakinankaratra, Vatovavy Fitovinany) des 22 régions de Madagascar. Ces régions sont les zones d'intervention de l'USAID dans le pays, dans lesquelles œuvrent principalement les 2 organismes bilatéraux de 1'USAID, JSI/MAHEFA et MSH/MIKOLO. En outre, ces régions abritent 77.9\% (17 018 871/21 842 167) de la population nationale malgache (INSTAT Madagascar 2013b). Cette étude entre dans le cadre de l'évaluation initiale du programme MCSP (survie de la mère et de l'enfant) de l'USAID à Madagascar. En effet, ce programme fournira un appui technique au Ministère de la Santé pour la réduction de la mortalité maternelle et néonatale à travers l'amélioration de la qualité des offres de services en santé et en mettant en œuvre des interventions basées sur l'évidence tout au long du continuum de soins, de la grossesse jusqu'à la période post-partum.

Cette étude quantitative transversale a été effectuée au niveau des établissements sanitaires publics. La liste des centres de santé de base (CSB) dans lesquels travaillent JSI/MAHEFA et MSH/MIKOLO, et la liste nationale des hôpitaux publics constituent les bases de sondage de notre échantillonnage. Les différents types d'établissements sanitaires publics existant dans le pays ont été inclus dans notre évaluation. Dans chaque région, deux centres de santé de base niveau 2 (CSB), un hôpital de référence et un hôpital universitaire ont été sélectionnés, à l'exception des régions de Boeny et Atsinanana avec l'ajout d'un CSB supplémentaire et la suppression d'un hôpital de référence de district (CHD). Comme seules les 6 régions contenant la capitale des ex-provinces du pays possèdent un hôpital universitaire (CHU), notre échantillon comprend $6 \mathrm{CHU}$. Pour ces 6 régions, 1 hôpital de référence de district (CHD) a également été inclus. Concernant les 9 autres régions, 1 hôpital de référence régional (CHRR) pour chaque région a été sélectionné et inclus dans notre échantillon. Par ailleurs, le critère de sélection des centres de santé de base ou CSB est leur proximité d'un hôpital de référence de district ou régional (CHD ou CHRR) qu'ils ne sont pas à plus de 50 $\mathrm{km}$ et à plus de deux heures de route en voiture . Ainsi, un échantillon représentatif de 52 établissements sanitaires publics, dont 6 hôpitaux universitaires CHU, 14 hôpitaux de référence ( $9 \mathrm{CHRR}$ au niveau régional et $5 \mathrm{CHD}$ au niveau district) ainsi que de 32 centres de santé de niveau primaire CSB a été enquêté pour refléter l'état des offres de service en santé maternelle et néonatale dans ces 15 régions de Madagascar. Cet échantillon a permis de répondre aux besoins d'information du programme et convient aux ressources allouées à MCSP Madagascar.
L'outil SARA (Service Availability and Readiness Assessment) developpé par l'OMS et l'USAID, destiné à fournir des informations essentielles sur l'état du système de santé à travers les indicateurs traceurs (O'Neill et al. 2013; WHO 2013), a été adapté aux besoins de cette évaluation. Durant la période de collecte des données sur le terrain, le formulaire SARA a été rempli à la fois sur tablette, avec la plateforme CommCare ODK (CommCare Open Data Kit, USA, www.commcarehq.org) et sur version papier. L'évaluation des ressources humaines dans les formations sanitaires a été effectuée au moyen d'un entretien avec le médecin chef et les personnels clés dans l'unité maternité des établissements sanitaires publics. La collecte de données sur la section et l'évaluation de la disponibilité et de la formation des agents de santé offrant les services de santé maternelle et néonatale, a duré approximativement une heure. La collecte sur terrain a été réalisée par 9 équipes enquêtrices comprenant chacune 1 médecin superviseur et 3 sages-femmes; elle a duré 3 semaines au cours du mois de septembre 2014 .

Etant donné que cette évaluation ne se porte pas sur des sujets humains, l'approbation d'un comité d'éthique n'a pas été requise. Néanmoins, une autorisation du Ministère de la Santé a été obtenue pour la conduite de cette évaluation dans les 15 régions.

A chaque fin de journée, les données saisies sur tablette ont été synchronisées avec le serveur cloud de CommCare ODK par chaque superviseur des équipes. Et le lendemain matin, le spécialiste des données les récoltait sous format Excel (Microsoft, USA). Ces données ont été ensuite compilées et analysées avec le SPSS20.0 (SPSS Inc, Chicago) selon le type d'établissement sanitaire (centre primaire CSB, hôpital de référence $\mathrm{CHRR} / \mathrm{CHD}$ et hôpital universitaire $\mathrm{CHU}$ ). Cette méthode d'analyse permet de considérer les différences dans les normes des agents de santé appliquées dans le pays pour chaque type d'établissement sanitaire, telles que le gynécoobstétricien, l'anesthésiste et le technicien de laboratoire exerçant uniquement dans les hôpitaux et inversement, les agents communautaires travaillant uniquement au niveau des CSB et pareillement, le pharmacien au niveau du CHU qui pourrait être un dispensateur de médicaments au niveau des centres primaires. Cette étude a comme intérêt principal, d'évaluer la disponibilité des agents de santé qualifiés offrant les services de santé maternelle et néonatale, selon l'outil de collecte SARA. Ainsi les agents de santé, ayant déclaré avoir reçu une formation ou un recyclage depuis 3 ans et plus, ne sont pas considérés comme qualifiés.

\section{Résultats}

\section{Caractéristiques générales de l'échantillon}

Les 52 établissements sanitaires publics évalués comprennent tous les types d'établissements sanitaires réalisant des accouchements dans le pays. Les structures sanitaires évaluées étaient composées de $62 \%(32 / 52)$ de centres de santé de base, de $17 \%(9 / 52)$ d'hôpitaux de référence au 
TABLEAU 1: Caractéristiques générales des 52 établissements sanitaires publiques évaluées, Madagascar 2014

\begin{tabular}{|c|c|c|c|c|}
\hline \multirow[t]{2}{*}{ Caractéristiques } & $\mathrm{CHU}(\%)$ & CHRR (\%) & CHD (\%) & CSB $(\%)$ \\
\hline & $N=6$ & $N=9$ & $N=5$ & $N=32$ \\
\hline \multicolumn{5}{|l|}{ Zone } \\
\hline Urbaine & 100 & 100 & 60 & 9 \\
\hline Rurale & 0 & 0 & 40 & 91 \\
\hline \multicolumn{5}{|c|}{ Consultation externe seulement } \\
\hline Oui & 0 & 0 & 0 & 13 \\
\hline Non & 100 & 100 & 100 & 87 \\
\hline \multicolumn{5}{|c|}{ Service Consultation Prénatale } \\
\hline Oui & 67 & 44 & 80 & 100 \\
\hline Non & 33 & 56 & 20 & 0 \\
\hline \multicolumn{5}{|c|}{ Service Accouchement } \\
\hline Oui & 100 & 100 & 100 & 100 \\
\hline Non & 0 & 0 & 0 & 0 \\
\hline \multicolumn{5}{|c|}{ Service Césarienne } \\
\hline Oui & 100 & 100 & 60 & 0 \\
\hline Non & 0 & 0 & 40 & 100 \\
\hline
\end{tabular}

CHU, Hôpitaux universitaires, $\mathrm{CHRR} / \mathrm{CHD}$, Hôpitaux de référence au niveau régional/district, CSB, Centres de Santé de Base.

niveau régional, de $11 \%$ (6/52) d'hôpitaux universitaires, et de $10 \%(5 / 52)$ d'hôpitaux de référence au niveau district. La collecte des données sur les agents de santé a été faite dans tous ces établissements sanitaires, et leurs principales caractéristiques sont présentées dans le Tableau 1. La majorité (91\%) des centres de santé primaire est localisée dans les zones rurales, tandis que les hôpitaux de niveau supérieur, universitaire et de référence régionale se trouvent dans les zones urbaines. Seulement $13 \%$ des CSB ne disposaient pas de lits d'hospitalisation pour les patientes dans leur centre sanitaire. Tous les centres de santé de base fournissent systématiquement des services de consultation prénatale mais certains hôpitaux n'en fournissent pas (33\% des CHU, $56 \%$ des CHRR et $20 \%$ des CHD). Tous les établissements sanitaires offrent des services d'accouchement. Par ailleurs, $40 \%$ des hôpitaux de référence au niveau district n'avaient pas de service pour l'opération césarienne fonctionnelle au moment de l'enquête, contrairement à tous les autres hôpitaux.

\section{Disponibilité des agents de santé dans les établissements sanitaires évalués}

$\mathrm{Au}$ niveau des centres de santé primaire, si le nombre médian de médecins généralistes était de 1,22\% des centres CSB rapportaient ne pas en avoir. Il en est de même pour les sages-femmes dont le nombre maximal au niveau d'un CSB s'élevait au nombre de 7, alors que certains CSB déclaraient ne pas en posséder dans leur personnel. Toutefois, si certains CSB avaient 17 agents communautaires qui leur étaient rattachés, quelques-uns disaient ne pas en avoir tandis que d'autres rapportaient en posséder 70 rattachés à leur niveau. En outre, concernant les hôpitaux de référence, le médecin généraliste manquait également pour $14 \%$ des CHRR/CHD alors que le nombre médian de médecins était de 3, et au maximum il y avait 9 médecins dans ces établissements. Pour l'opération césarienne, l'anesthésiste, qu'il soit un infirmier ou un médecin, et le gynécologue obstétricien pouvaient parfois faire défaut dans les hôpitaux de référence.
Néanmoins, le nombre médian de sage-femme était de 6 au niveau des hôpitaux de référence de premier niveau (CHRR et CHD) de notre échantillon. Par ailleurs, 1 technicien de laboratoire et 1 pharmacien en général, offraient leur service pour la santé maternelle et néonatale au niveau de ces hôpitaux. En revanche, pour les hôpitaux universitaires, on ne manquait pas de personnel clé pour offrir les services en santé maternelle et néonatale, et les nombres médians étaient de 6 médecins généralistes, 26 sages-femmes et 2 infirmiers. Quant à l'offre d'opération césarienne dans ces $\mathrm{CHU}$, ils disposaient en général de 2 anesthésistes et de 1 gynécologue obstétricien (Tableau 2).

\section{Disponibilité de personnels qualifiés ayant eu une formation récente en santé maternelle et néonatale dans les établissements sanitaires publics}

Dans cette évaluation, un agent de santé qualifié rapportait avoir reçu au cours des 2 dernières années, une formation relative au domaine de la santé de la mère et de l'enfant. Les thèmes sur lesquels les agents de santé ont été formés incluent la consultation prénatale focalisée, la santé de la mère et du nouveau-né, les soins obstétricaux et néonataux d'urgence (SONU), la consultation post-natale, l'audit du décès maternel et les soins humanisés.

Parmi les médecins généralistes qualifiés travaillant dans les établissements sanitaires évalués, la grande majorité $(49 \%)$ provenaient des centres de santé de niveau primaire. Le tiers des médecins évalués ayant reçu des mises à jour sur la santé maternelle et néonatale exerçaient dans les hôpitaux de référence au niveau régional et au niveau district. En revanche, les médecins des hôpitaux universitaires constituaient la faible proportion de ces personnels ayant reçu une formation récente en santé maternelle et néonatale. La disponibilité des médecins qualifiés est statistiquement indépendante du type d'établissement sanitaire public $(p=0.29)$. Quant aux sages-femmes, contrairement aux médecins, c'étaient celles exerçant dans les centres de santé primaire qui ont été les moins nombreuses à être formées (21\%) et les plus nombreuses provenaient des hôpitaux universitaires (53\%). Le quart des sages-femmes ayant reçu une mise à jour de leurs connaissances et compétences techniques provenait des hôpitaux de référence tant au niveau district que de la région (26\%). Les sages-femmes qualifiées et le type d'établissement sanitaire sont également statistiquement indépendants $(p=0.38)$. Concernant les infirmiers ayant reçu une formation en santé maternelle et néonatale, la moitié travaillait dans les hôpitaux de référence (CHRR/CHD), 40\% travaillaient dans les hôpitaux universitaires et seulement $10 \%$ provenaient des centres de santé primaire. Comme les gynécologues obstétriciens exercent uniquement au niveau des hôpitaux, les données sur les récentes mises à jour de leurs compétences techniques en santé maternelle et néonatale ont été collectées au niveau des hôpitaux de référence (CHRR/CHD) et des hôpitaux universitaires (CHU). La majorité de ces gynécologues obstétriciens provenaient des hôpitaux universitaires (67\%) 
TABLEAU 2: Nombre médian des agents de santé par qualification et par type d'établissement sanitaire, Madagascar 2014.

\begin{tabular}{|c|c|c|c|c|c|c|}
\hline \multirow[t]{2}{*}{ Variable } & \multicolumn{2}{|r|}{$\mathrm{CHU}$} & \multicolumn{2}{|c|}{ CHRR/CHD } & \multicolumn{2}{|r|}{ CSB } \\
\hline & Médiane & Minimum; Maximum & Médiane & Minimum; Maximum & Médiane & Minimum; Maximum \\
\hline \multicolumn{7}{|l|}{ Personnels médicaux } \\
\hline Médecin généraliste & 6 & $2 ; 33$ & 3 & $0 ; 9$ & 1 & $0 ; 4$ \\
\hline Anesthésiste & 2 & $1 ; 7$ & 1 & $0 ; 3$ & NA & NA \\
\hline Gynécologue Obstétricien & 1 & $1 ; 32$ & 1 & $0 ; 1$ & NA & NA \\
\hline Sage-femme & 26 & $7 ; 78$ & 6 & $2 ; 10$ & 1 & $0 ; 7$ \\
\hline Infirmier & 2 & $1 ; 89$ & 2 & $1 ; 18$ & 0 & $0 ; 3$ \\
\hline Pharmacien & 2 & $0 ; 3$ & 1 & $0 ; 4$ & NA & NA \\
\hline Technicien de laboratoire & 1 & $1 ; 5]$ & 1 & $0 ; 5$ & NA & NA \\
\hline \multicolumn{7}{|l|}{ Personnels non-médicaux } \\
\hline Aide sanitaire & 0 & $0 ; 1]$ & 0 & $0 ; 2$ & 0 & $0 ; 1$ \\
\hline Agent d'appui & 2 & $1 ; 56]$ & 1 & $0 ; 10$ & 0 & $0 ; 3$ \\
\hline Agents Communautaires & NA & NA & NA & NA & 17 & $0 ; 70$ \\
\hline
\end{tabular}

CHU, Hôpitaux universitaires, CHRR/CHD, Hôpitaux de référence au niveau régional/district, CSB, Centres de Santé de Base, NA, N’est pas applicable.

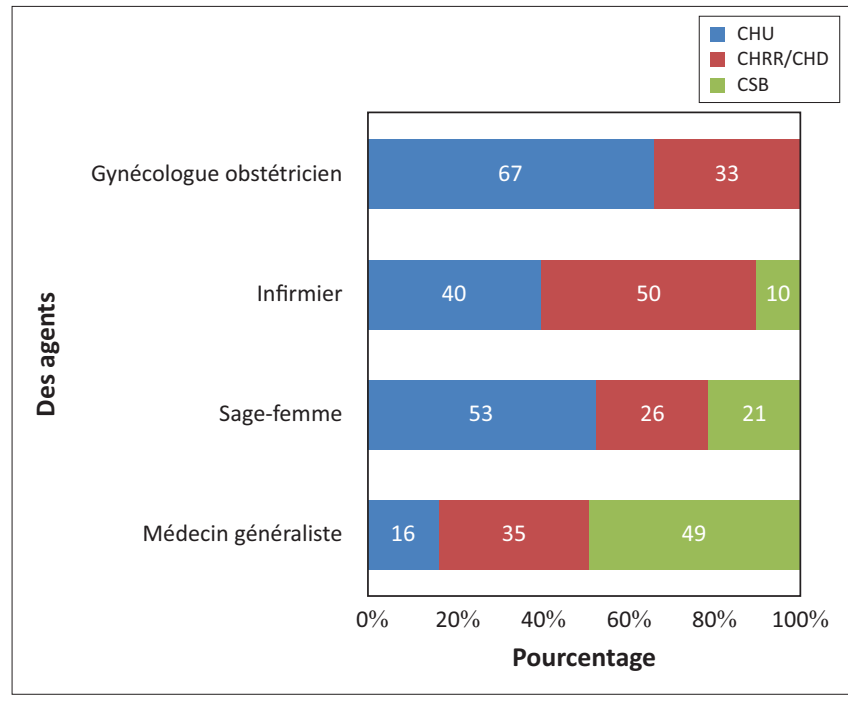

CHU, Hôpitaux universitaires, CHRR/CHD, Hôpitaux de référence au niveau régional/district, CSB, Centres de Santé de Base.

FIGURE 1: Distribution des agents de santé ayant reçu une formation en santé maternelle et néonatale lors des 2 dernières années dans les établissements sanitaires des 15 régions, Madagascar 2014

et les 33\% restants travaillaient dans les hôpitaux de référence au niveau régional et au niveau district (Figure 1).

La disponibilité de ressources humaines qualifiées, ayant eu une mise à jour récente de leurs connaissances et compétences techniques sur la santé de la mère et de l'enfant, varie de $0 \%$ (Sava, Melaky) à 92\% (Vatovavy Fitovinany) dans les différentes régions évaluées. Seules 3 régions (Atsimo Andrefana, Boeny et Vatovavy Fitovinany) présentent une proportion élevée $(>80 \%)$ de prestataires de santé ayant reçu une récente mise à jour technique en santé maternelle et néonatale. Cinquante trois pourcent $(8 / 15)$ des régions de notre échantillon avait une très faible $(<20 \%)$ disponibilité de ressources humaines qualifiées ayant eu une mise à jour de leurs compétences techniques, pour offrir les services de santé maternelle et néonatale dans les établissements sanitaires. Les 4 autres régions (Diana, Sofia, Vakinankaratra et Haute Matsiatra) avaient de $21 \%$ à $40 \%$ de leurs ressources humaines qui avaient reçu une formation ou une mise à jour récente de leurs connaissances et compétences techniques en santé de la mère et de l'enfant (Figure 2).

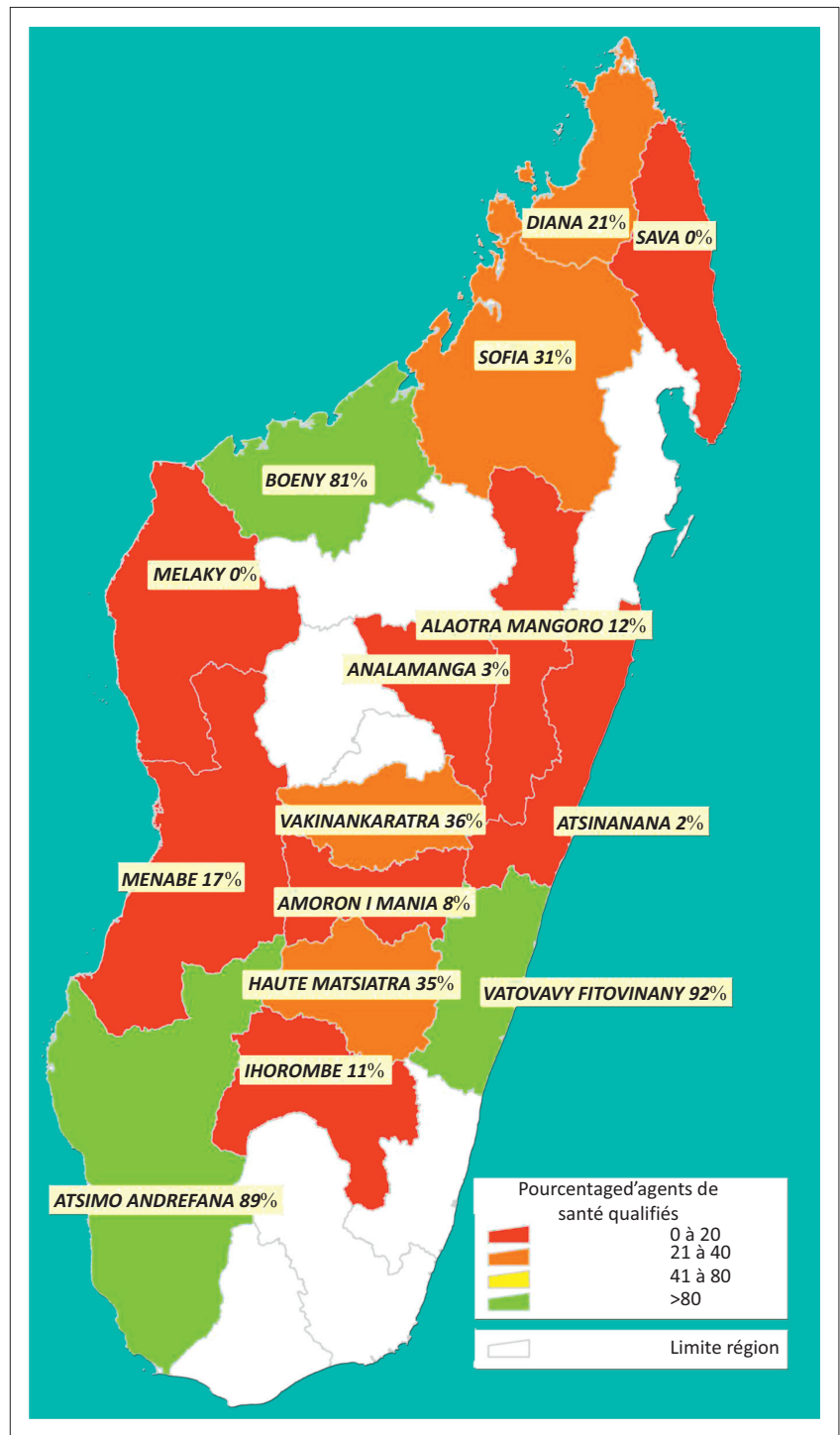

FIGURE 2: Répartition des ressources humaines qualifiées en santé maternelle et néonatale dans les 15 régions enquêtées, Madagascar 2014.

\section{Discussion}

Cette étude rapporte que le nombre médian de médecins variait de $1(\mathrm{CSB}), 3(\mathrm{CHRR} / \mathrm{CHD})$ à $6(\mathrm{CHU})$ tandis que celui des sages-femmes variait de 1 (CSB), 6 (CHRR/CHD) 
à 26 dans les CHU. En revanche, le nombre médian de gynécologues obstétriciens au niveau des hôpitaux était de 1 , alors que certains hôpitaux de référence n'avaient pas de gynécologue obstétricien pour assurer les opérations césariennes.

Concernant les formations récentes dans le domaine de la santé de la mère et de l'enfant, la distribution des personnels qualifiés variait selon le type d'établissement sanitaire (CSB, $\mathrm{CHRR/CHD}$ et $\mathrm{CHU}$ ). Les deux-tiers des gynécologues obstétriciens et plus de la moitié des sages-femmes, ayant reçu une formation au cours des 2 dernières années, exerçaient au niveau des hôpitaux universitaires. Par contre, la moitié des médecins formés en santé maternelle et néonatale travaillaient dans les centres de santé de base. De plus, la disponibilité des ressources humaines qualifiées, ayant eu une mise à jour récente sur leurs connaissances et compétences techniques sur la santé de la mère et de l'enfant, variait de $0 \%$ à $92 \%$ dans les différentes régions évaluées. Sur les 15 régions, huit avaient une très faible proportion (<20\%) d'agents de santé qui avaient reçu une récente formation en santé maternelle et néonatale. Seules les 3 régions, Atsimo Andrefana, Boeny et Vatovavy Fitovinany ont bénéficié de récentes formations sur la santé de la mère et de l'enfant parmi les 15 régions évaluées, probablement dû à l'existence de partenaires techniques qui n'ont pas cessé leur appui durant la récente crise socio-politique dans le pays.

Ces résultats mettent en exergue d'une part la déficience en ressources humaines dans les établissements sanitaires publics, et la carence en formation sur les récentes mises à jour des connaissances et compétences en santé maternelle et néonatale d'autre part. Pourtant, ces problèmes de pénurie de prestataires de santé sont largement communs aux pays d'Afrique qui ont en moyenne 2.6 médecins et 12 infirmières ou sages-femmes pour servir 10000 personnes (INSTAT Madagascar 2013b). Dans le cas de Madagascar, cette densité descend encore à 1.6 médecin pour 10000 personnes (INSTAT Madagascar 2013b).

En outre, les prestataires de santé disponibles dans les centres sanitaires pour les soins de la mère et de l'enfant manquent souvent également de formation et de mise à jour de leurs connaissances et compétences techniques (WHO 2014b). Pour y remédier, la délégation de tâches à des prestataires de santé moins qualifiés (Niango et al. 2010) représente une des stratégies appliquées pour permettre une offre de soins maternels et néonatals, surtout dans les établissements manquant de personnel qualifié (Dovlo 2004). A titre d'illustration, les sages-femmes sont déjà autorisées à administrer du sulfate de magnésium dans les établissements sanitaires à Madagascar (Jennings et al. 2011). Une autre stratégie est d'intégrer les agents communautaires dans le système de santé, recommandée notamment dans les pays en cours de développement (Fujioka \& Smith 2011). $\mathrm{Au}$ cours des dernières années, Madagascar a établi une politique de santé communautaire qui vise à améliorer l'offre de services en santé à travers les agents communautaires rattachés au niveau des centres de santé primaires. Et récemment, le Ministère de la Santé en collaboration avec des partenaires techniques comme l'UNICEF et l'UNFPA a contractualisé des paramédicaux pour répondre aux besoins importants de ressources humaines au niveau des centres de santé de base.

Cependant, le renforcement en effectif des prestataires de santé ne suffit pas pour offrir les services de santé. En effet, le renforcement en capacité de ces prestataires, spécifiquement dans le domaine de la santé maternelle et néonatale serait primordial car un personnel qualifié serait en mesure de répondre aux besoins en soins maternels vitaux pour la réduction effective de la mortalité maternelle (Zulu et al. 2014). Comme nous le démontrent les résultats de cette évaluation, la disponibilité des agents de santé qualifiés offrant les services de santé maternelle et néonatale est assez faible, inférieure à $20 \%$ pour 8 parmi les 15 régions évaluées. De même, le renforcement continu de la capacité des prestataires de santé aurait un impact positif sur la réalisation des interventions conduisant à l'amélioration de la santé maternelle et néonatale (Ariff et al. 2010; Zulu et al. 2014). Même si les activités de formation de mise à jour des compétences techniques des prestataires de santé font déjà partie d'une des priorités actuelles du Ministère de la Santé et de toutes les parties prenantes de la santé maternelle et néonatale, les résultats de cette évaluation démontrent une grande lacune dans le renforcement des capacités des agents de santé dans les établissements sanitaires publics.

Afin d'optimiser les efforts du pays dans la réduction de la mortalité maternelle et néonatale, il est important que les efforts du Ministère de la Santé et de tous les partenaires techniques et financiers soient coordonnés et renforcés. Le volet des ressources humaines qualifiées pour prodiguer les offres de services de santé devrait être une des priorités des stratégies pour l'accélération et la continuité de la réduction de la mortalité maternelle et néonatale, même si les objectifs 4 et 5 du millénaire pour le développement ne seront pas atteints pour Madagascar.

Ultérieurement, cette étude pourrait être étendue aux 7 autres régions du pays afin d'obtenir un tableau de la disponibilité des agents de santé qualifiés pour assurer les services de santé maternelle et néonatale dans les établissements sanitaires publics à l'échelle nationale. De plus, une étude sur l'observation des soins réalisées par les agents de santé dans ces établissements sanitaires pourrait également compléter cette présente évaluation. En outre, la conduite d'une évaluation de la compétence technique en santé maternelle et néonatale des agents de santé exerçant dans les établissements sanitaires publics qui montrerait les besoins réels en formation (Ariff et al. 2010), serait idéale pour appuyer la stratégie de renforcement de la capacité technique du personnel de santé à l'échelle nationale. En effet, l'augmentation de la disponibilité des agents de santé qualifiés en santé maternelle et néonatale dans 
les établissements sanitaires publics serait un atout pour Madagascar dans son effort continu pour la réduction de la mortalité maternelle et infantile.

L'échantillonnage des sites évalués constitue une limite importante dans cette étude. En effet, les sites sont tous localisés dans les 15 régions d'intervention de l'USAID et leur sélection raisonnée dans les bases de sondage dépendait de leur proximité d'un hôpital de référence. Néanmoins, cette évaluation couvre tous les niveaux d'établissements sanitaires du système public, allant des hôpitaux universitaires, des hôpitaux de référence de la région et des districts jusqu'aux centres de santé de base. Par ailleurs, cette étude n'a pas réalisé une évaluation des connaissances techniques des prestataires de santé, ni de leurs aptitudes à offrir les services aux clients, comme il a été fait précédemment (Banchani \& Tenkorang 2014; Bazant et al. 2013; Ranjalahy et al. 2011). Cette évaluation a permis d'avoir un aperçu de la disponibilité des prestataires de santé qualifiés offrant les services de santé maternelle et néonatale dans les 15 régions, mais ne serait pas généralisable au niveau national sans la conduite d'autres études complémentaires, notamment l'évaluation de la connaissance et de la compétence technique des agents de santé dans le domaine de la mère et de l'enfant (Ariff et al. 2010).

\section{Conclusion}

L'objectif de cette étude était d'évaluer la disponibilité des agents de santé qualifiés pouvant offrir les services de santé maternelle et néonatale dans les établissements sanitaires publics. L'outil SARA de l'OMS a servi pour la collecte de données quantitatives sur les ressources humaines et leur formation récente en santé maternelle et néonatale au cours des 2 dernières années, auprès des 52 établissements sanitaires publics comprenant 6 hôpitaux universitaires, 14 hôpitaux de référence au niveau régional et au niveau district ainsi que de 32 centres de santé de base. Une importante pénurie d'agents de santé qualifiés, avec une récente mise à jour dans le domaine de la santé de la mère et de l'enfant a été constatée dans tous les niveaux d'établissements sanitaires publics. En ayant mis en exergue la faiblesse des ressources humaines qualifiées, pour offrir les services de santé maternelle et néonatale dans les établissements sanitaires publics, à la veille de 2015, cette évaluation trouve son importance en rapportant l'un des facteurs clés expliquant l'échec de l'atteinte des objectifs 4 et 5 de l'OMD de Madagascar, en dépit des différentes stratégies et des activités multiples déjà réalisées dans tout le pays.

\section{Remerciements}

Nous remercions le Ministère de la Santé de Madagascar pour son appui et pour nous avoir autorisés à réaliser cette évaluation auprès des établissements sanitaires publics. Nos remerciements s'adressent également à toutes les directions régionales de la santé, à tous les services de district de la santé, à tous les personnels des établissements sanitaires évalués ainsi qu'aux équipes enquêtrices. Que toute l'équipe de Jhpiego au siège central et toute l'équipe à Madagascar, qui n'ont pas ménagé d'efforts dans la réalisation de cette évaluation trouvent ici la reconnaissance de leur travail.

\section{Financement}

L'USAID a financé cette évaluation sous l'accord de coopération AID- OAA -A- 14-00028. Son contenu est la responsabilité du Programme USAID/MCSP (Maternal and Child Survival Program) et ne reflète pas nécessairement les points de vue de l'USAID ou du Gouvernement des ÉtatsUnis.

\section{Les contributions des auteurs}

Chacun des auteurs a contribué activement à la réalisation de cette évaluation, du développement du protocole, de la mise en œuvre sur terrain, des analyses des données jusqu'à l'élaboration du rapport final de cette étude. Cet article a été approuvé par tous les auteurs.

\section{Références}

Anand, S. \& Bärnighausen, T., 2004, 'Human resources and health outcomes: Crosscountry econometric study', Lancet 364(9445), 1603-1609. PMID: 15519630 http://dx.doi.org/10.1016/S0140-6736(04)17313-3

Ariff, S., Soofi, S.B., Sadiq, K., Feroze, A.B., Khan, S., Jafarey, S.N. et al., 2010, 'Evaluation of health workforce competence in maternal and neonatal issues in public health sector of Pakistan: An assessment of their training needs', BMC Health Services Research 10, 319. PMID: 21110888, http://dx.doi.org/10.1186/1472-6963-10-319

Banchani, E. \& Tenkorang, E.Y., 2014, 'Implementation challenges of maternal health care in Ghana: The case of health care providers in the Tamale Metropolis', BMC Health Services Research 14, 7. PMID: 24393358, http://dx.doi.org/10.1186/14726963-14-7

Bazant, E., Rakotovao, J.P., Rasolofomanana, J.R., Tripathi, V., Gomez, P., Favero, R. et al., 2013, 'Qualité des soins pour prévenir et traiter l'hémorragie du postpartum et la pré-éclampsie/éclampsie: une évaluation fondée sur l'observation dans les hôpitaux de Madagascar', Médecine et santé tropicales 23(2), 168-175. PMID: 23694783, http://dx.doi.org/10.1684/mst.2013.0161

Chen, L., Evans, T., Anand, S., Boufford, J.I., Brown, H., Chowdhury, M. et al., 2004 'Human resources for health: Overcoming the crisis', Lancet 364, 1984-1990. PMID: 15567015, http://dx.doi.org/10.1016/S0140-6736(04)17482-5

Dovlo, D., 2004, 'Using mid-level cadres as substitutes for internationally mobile health professionals in Africa. A desk review', Human Resources for Health 2(1), 7. PMID: 15207010, http://dx.doi.org/10.1186/1478-4491-2-7

Fujioka, A. \& Smith, J., 2011, Prevention and management of postpartum hemorrhage and pre-eclampsia/eclampsia: National programs in selected USAID program-supported countries. Maternal and Child Health Integrated Program MCHIP, Jhpiego, Baltimore.

Fulton, B.D., Scheffler, R.M., Sparkes, S.P., Auh, E.Y., Vujicic, M. \& Soucat, A., 2011, 'Health workforce skill mix and task shifting in low income countries: A review of recent evidence', Human Resources for Health 9, 1. PMID: 21223546, http:// dx.doi.org/10.1186/1478-4491-9-1

Gerein, N., Green, A. \& Pearson, S., 2006, 'The implications of shortages of health professionals for maternal health in sub-Saharan Africa', Reproductive Health Matters 14(27), 40-50. PMID: 16713878, http://dx.doi.org/10.1016/S09688080(06)27225-2

Harimanana, A., Barennes, H. \& Reinharz, D., 2011, 'Organizational analysis of maternal mortality reduction programs in Madagascar', The International Journal of Health Planning and Management 26, e186-e196. PMID: 21796678, http:// dx.doi.org/10.1002/hpm.1077

INSTAT Madagascar, 2013a, Enquête nationale sur le suivi des objectifs du millénaire pour le développement à Madagascar. Disponible au site internet: http://www. instat.mg/pdf/ensomd-2012-2013_resume.pdf [consulté le 03/10/2014].

INSTAT Madagascar, 2013b, Madagascar en chiffres, population et démographie. Disponible au site internet: http://www.instat.mg/index.php?option=com content\&view=article\&id=33\&ltemid=56 [consulté le 10/02/2015].

Jennings, L., Yebadokpo, A.S., Affo, J., Agbogbe, M. \& Tankoano, A., 2011, 'Task shifting in maternal and newborn care: A non-inferiority study examining delegation of antenatal counseling to lay nurse aides supported by job aids in Benin Implementation Science 6, 2. PMID: 21211045, http://dx.doi.org/10.1186/17485908-6-2

MCHIP Madagascar-Jhpiego, 2011, Quality of care of the prevention and management of common maternal and newborn complications in health facilities in Madagascar. Jhpiego, Baltimore. Disponible au site internet: http://www. Madagascar. Jhpiego, Baltimore. Disponible au site internet: http://www. [consulté le 03/10/2014]. 
Narasimhan, V., Brown, H., Pablos-Mendez, A., Adams, O., Dussault, G., Elzinga, G. et al., 2004, 'Responding to the global human resources crisis', Lancet 363 et al., 2004, 'Responding to the global human resources crisis', Lancet 363,

Niango, D.D., Mutihir, J.T., Laabes, E.P., Kigbu, J.H. \& Buba, M., 2010, 'Skilled attendance: The key challenges to progress in achieving MDG-5 in North Central Nigeria', African Journal of Reproductive Health 14(2), 129-138. PMID: 21243925.

O'Neill, K., Takane, M., Sheffel, A., Abou-Zahr, C., Boerma, T., 2013, 'Monitoring service delivery for universal health coverage: The service availability and readiness assessment', Bulletin of the World Health Organization 91, 923-931. PMID: 24347731, http://dx.doi.org/10.2471/BLT.12.116798

Ranjalahy, R.J., Bazant, E., Rakotovao, J.P., Cantor, D., Jeng-Joof, Y., Noeliarivelo, A \& Ribaira, G.J., 2011, Evaluation de la qualité des soins maternels et néonatals à Madagascar, Maternal and Child Health Integrated Program (MCHIP), Johns Hopkins University, TANDEM, Jhpiego, Baltimore.

The Lancet Commissions, 2010, 'The millennium development goals: A cross-sectoral analysis and principles for goal setting after 2015', Lancet 376, 991-1021.

UNICEF, 2009, Pallier la pénurie de personnel de santé: une tâche prioritaire pour améliorer la santé de la mère et du nouveau-né. Disponible au site internet: http://www.unicef. org/french/sowc09/docs/SOWC09-Encadres-2.3-FR.pdf [consulté le 22/02/2015].
WHO, 2006, The world health report 2006: Working together for health. World Health Organization, Geneva. Disponible au site internet: http://www.who.int/ whr/2006/whr06_en.pdf?ua=1 [consulté le 22/02/2015].

WHO, 2013, Service availability and readiness assessment, an annual monitoring system for service delivery. Reference Manual, version 2.1. World Health
Organization, Geneva. Disponible au site internet: http://www.who.int/ healthinfo/systems/SARA_Reference_Manual_Full.pdf [consulté le 20/08/2014].

WHO, 2014a, Maternal mortality, factsheet 348, updated May 2014. World Health Organization, Geneva. Disponible au site internet: http://www.who.int/ mediacentre/factsheets/fs348/en/ [consulté le 22/02/2015].

WHO, 2014b, World health statistics 2014. 1. Health status indicators. 2. World health. 3. Health services - Statistics. 4. Mortality. 5. Morbidity. 6. Life expectancy. 7. Demography. 9. Statistics. 10. World Health Organization. World Health Organization, Geneva. Disponible au site internet; http://apps.who.int/iris/bitstre am/10665/112738/1/9789240692671_eng.pdf [consulté le 08/02/2015].

Zulu, M.J., Kinsman, J., Michelo, C. \& Hurtig, A.K., 2014, 'Integrating national community-based health worker programmes into health systems: A systematic review identifying lessons learned from low and middle income countries', BMC Public Health 14, 987. PMID: 25245825, http://dx.doi.org/10.1186/1471-245814-987 